\title{
Optimum Power Allocation for Sum Rate Improvement in AF Multi-way Relay Networks
}

\author{
Shama N. Islam, Salman Durrani and Parastoo Sadeghi \\ Research School of Engineering \\ College of Engineering and Computer Science (CECS) \\ The Australian National University (ANU) \\ Canberra 0200 ACT, Australia \\ E-mail: shama.islam@anu.edu.au, salman.durrani@anu.edu.au, parastoo.sadeghi@anu.edu.au
}

\begin{abstract}
In this paper, we formulate and study the optimum power allocation problem in an amplify and forward (AF) multiway relay network (MWRN). We derive closed-form analytical expressions for the optimum power allocation coefficients for the data power and the pilot power of the users and the relay to maximize the sum rate. We assume that only long term statistical channel state information (CSI) (i.e., channel variance) are available to each user and the relay. Our results show that to achieve the same sum rate, optimum power allocation requires $7-9$ dB less transmit power compared to equal power allocation depending on users' channel conditions. In addition, we show that for optimum power allocation, the reduction in the sum rate due to channel estimation errors, becomes less compared to the case of equal power allocation.
\end{abstract}

Index Terms-multi-way relay network, amplify and forward, power allocation, sum rate, channel state information.

\section{INTRODUCTION}

Multi-way relay networks (MWRNs) [1]-[6] have recently been proposed to realize the well-established larger capacity and spectral efficiency benefits of two-way relay networks (TWRNs) [7]-[9] in a multi-user scenario. In a MWRN, all users exchange their information with each other through a single relay without any direct link between them. MWRNs have important potential applications like teleconferencing, message exchange in a social network or satellite communication [1], [4].

In this paper, we study amplify and forward (AF) MWRNs for their simpler implementation and lower complexity at the relay. Recent studies on AF MWRNs include error performance analysis [4], [10] and outage probability and sum rate analysis [4], [11] for the cases when users can transmit in a pairwise or a non-pairwise manner. Non-pairwise transmission strategies can ensure higher multiplexing gain, though these strategies require beamforming [12] or precoding [4] at the relay which adds to the signal processing complexity of the system. Hence, in this paper, we focus on the pairwise transmission which involves less signal processing complexity at the relay. In a pairwise transmission based MWRN, the $\ell^{t h}$ and the $(\ell+1)^{t h}$ users form a pair at the $\ell^{t h}$ time slot, where $\ell \in[1, L-1]$ and $L$ is the number of users in the MWRN.

Recently, optimum power allocation between the relay and user power and between the data and pilot powers has been investigated for TWRNs for rate adaptation [13], rate fairness [14], maximization of the signal-to-noise (SNR) ratio [15], minimization of the mean square error of the channel estimation [16] or minimization of the asymptotic symbol error rate [17], respectively. Moreover, power allocation has been studied to maximize the sum rate of all the users in a multi-user TWRN [18], [19], which is a special class of MWRNs, where each user exchanges message only with its pre-defined partner. In the aforementioned studies on MWRNs, full instantaneous channel state information (CSI) is assumed to be available to all the users and the relay. However, power allocation strategies in a more practical scenario, where only statistical CSI is available to each user and the relay, has not yet been investigated.

Based on the above open problems, we make the following contributions in this paper:

- We derive the expressions for the optimum power allocation coefficients to maximize the achievable sum rate in an AF MWRN assuming that only statistical CSI is available at each user and the relay. Numerical simulation results verify the correctness of the analytical derivations.

- We observe that to achieve the same sum rate, optimum power allocation requires $7-9 \mathrm{~dB}$ less power compared to equal power allocation depending upon users' channel conditions.

- We show that compared to equal power allocation, optimum power allocation helps to lessen the impact of imperfect CSI on the achievable sum rate.

The rest of the paper is organized in the following manner. The system model of an AF MWRN is presented in Section II. The pilot transmission and the data transmission are discussed in Section III and Section IV, respectively. The sum rate analysis is provided in Section V. The optimum power allocation coefficients to maximize the sum rate are derived in Section VI. Section VII provides the simulation results for verification of the analytical solutions. Finally, conclusions are provided in Section VIII.

\section{SySTEM MODEL}

We consider an $L$-user AF MWRN where the users exchange their information through a single relay, as illustrated 


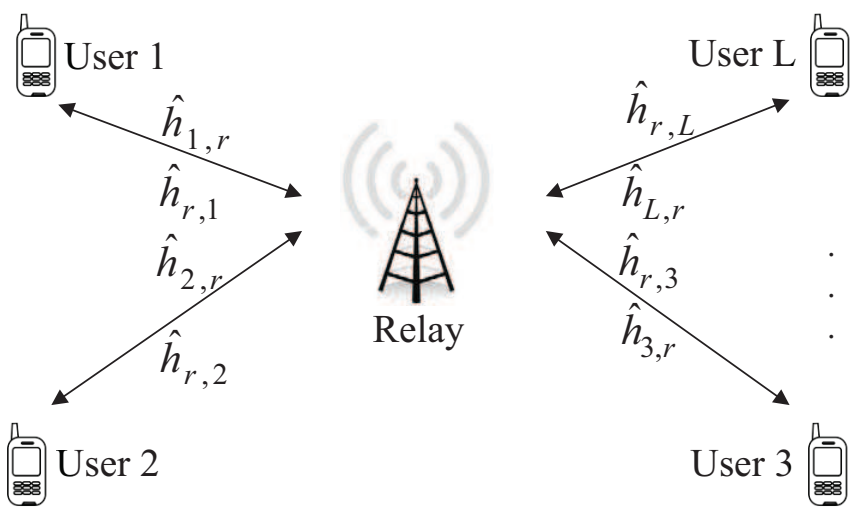

Fig. 1. System model for an $L$-user multi-way relay network (MWRN), where the users exchange information with each other via the relay $R$.

\section{in Fig. 1.}

We assume that the users transmit in a half-duplex manner and do not have any direct links between them. The communication among the users takes place in two phases-multiple access and broadcast phase. In the multiple access phase, the users transmit their data simultaneously in a pairwise manner and the relay receives the sum of the signals. That is, at the $\ell^{t h}$ time slot, the $\ell^{t h}$ and the $(\ell+1)^{t h}$ users transmit simultaneously, where $\ell \in[1, L-1]$. In the broadcast phase, the relay broadcasts the amplified network coded signal to all the users. Thus, a total number of $2(L-1)$ time slots are needed for all the users to extract the network coded messages corresponding to each user pair. Throughout each round of communication (i.e., $2(L-1)$ time slots), each user transmits a signal packet of $T$ data transmission blocks and the relay transmits $(L-1)$ signal packets, each of $T$ data transmission blocks, where each transmission block is of length $T^{\prime}$. We choose the index for time slot as $t_{s}$ and the signal block index as $t$ where, $t_{s} \in[1, L-1]$ and $t \in[1, T]$.

At the $t_{s}^{t h}$ time slot, the channel from the $j^{\text {th }}$ user to the relay is denoted by $h_{j, r}^{t_{s}}$ and the channel from the relay to the $j^{\text {th }}$ user by $h_{r, j}^{t_{s}}$, where $j \in[1, L]$. We make the following assumptions regarding the channels:

1) The channels are assumed to be block Rayleigh fading reciprocal channels which remain constant during one signal packet transmission in a certain time slot in a certain multiple access or broadcast phase. The channels in different time slots are considered independent.

2) The channel coefficients are zero mean complex-valued Gaussian random variables with variances $\sigma_{h_{j, r}}^{2}=\sigma_{h_{r, j}}^{2}$.

3) The long term statistical CSI only, e.g., channel variances, are known at the users and the relay [20], [21].

In the next section, we discuss how each user estimates the channel coefficients through pilot signals.

\section{Pilot Transmission}

In an AF MWRN, each user needs to know the channel coefficients of all the other users to cancel self-interference components of the other users' signals in the signal received from the relay [10]. To accomplish this, each of the two simultaneously transmitting users transmit a pilot signal in separate pilot transmission blocks prior to data transmission in the multiple access phase. The relay amplifies and forwards this pilot signal to all the users in separate pilot transmission blocks of the broadcast phase. Then each user estimates the product of the channel coefficients of the transmitting users and the receiving user using minimum mean square error (MMSE) estimation. Such MMSE estimation methods are widely used in the literature of channel estimation [16], [20], [22]. Since the channel is constant during one signal packet transmission, only one pilot signal per data signal packet is required, provided the data and the pilot power are optimized.

At the $t_{s}^{\text {th }}$ time slot, we denote the power of the pilot signal at the users and at the relay as $P_{p, s}$ and $P_{p, r}$, respectively. Similarly, we denote the power of the data signal at the users and the relay at the $t_{s}^{t h}$ time slot by $P_{d}$ and $P_{r}$, respectively. We consider the average power per signal is $P$. Next, we discuss the pilot transmission protocols at the users and the relay. Since, we consider information exchange in a certain time slot, we can omit the superscript $t_{s}$ to simplify the notations.

\section{A. Pilot Transmission at the Users}

The $q^{t h}$ users transmits a pilot signal $X_{q}$ at the $t=q^{t h}$ pilot transmission block $\left(q=i, i+1\right.$ for $\left.t_{s}=i, i \in[1, L-1]\right)$ and the relay receives the signal

$$
R_{q}=\sqrt{P_{p, s}} h_{q, r} X_{q}+n_{p, 1} .
$$

We assume that $X_{q}=1$ and $n_{p, 1}$ is a zero mean complex valued AWGN with variance $\sigma_{n}^{2}=\frac{N_{0}}{2}$ per dimension.

\section{B. Pilot Transmission at the Relay}

Prior to data transmission in the broadcast phase, the relay amplifies the received pilot signals and forwards them to all the users. At the $t=q^{t h}$ pilot transmission block, the $j^{t h}$ $(j \in[1, L])$ user receives the signal

$$
Y_{q}=\alpha_{q} h_{r, j}\left(\sqrt{P_{p, s}} h_{q, r} X_{q}+n_{p, 1}\right)+n_{p, 2},
$$

where, $\alpha_{q}=\sqrt{\frac{P_{p, r}}{P_{p, s} \sigma_{h_{q, r}}^{2}+\sigma_{n}^{2}}}$ is the amplification factor and $n_{p, 2}$ is a zero mean complex valued AWGN with variance $\sigma_{n}^{2}$ per dimension. It can be noted that the relay do not estimate the users' channel coefficients by itself, rather it utilizes the channel variances to calculate the amplification factors.

Finally, the $j^{\text {th }}$ user performs MMSE estimation to obtain the estimate of the cascaded channel $h_{j, q}=h_{r, j} h_{q, r}$ as $\hat{h}_{j, q}$. We can model the channel $h_{j, q}$ as

$$
h_{j, q}=\hat{h}_{j, q}+\tilde{h}_{j, q},
$$

where $h_{j, q}$ is the estimated channel and $\tilde{h}_{j, q}$ represents the estimation error [22]. Thus, the user estimates:

$$
\begin{aligned}
\hat{h}_{j, q} & =E\left[h_{j, q} Y_{q}^{*}\right] E^{-1}\left[\left|Y_{q}\right|^{2}\right] Y_{q} \\
& =\frac{\alpha_{q} \sigma_{h_{q, r}}^{2} \sqrt{P_{p, s}} \sigma_{h_{r, j}}^{2}}{\alpha_{q}^{2} \sigma_{h_{q, r}}^{2} P_{p, s} \sigma_{h_{r, j}}^{2}+\alpha_{q}^{2} \sigma_{h_{r, j}}^{2} \sigma_{n}^{2}+\sigma_{n}^{2}} Y_{q}
\end{aligned}
$$


and the estimation error variance at the relay is:

$$
\begin{aligned}
\sigma_{\tilde{h}_{j, q}}^{2} & =E\left[\left|h_{q, r}\right|^{2}\right]-E\left[\left|\hat{h}_{q, r}\right|^{2}\right] \\
& =\frac{\sigma_{h_{q, r}}^{2} \sigma_{h_{r, j}}^{2}\left(\alpha_{q}^{2} \sigma_{h_{r, j}}^{2}+1\right) \sigma_{n}^{2}}{\alpha_{q}^{2} \sigma_{h_{q, r}}^{2} \sigma_{h_{r, j}}^{2} P_{p, s}+\left(\alpha_{q}^{2} \sigma_{h_{r, j}}^{2}+1\right) \sigma_{n}^{2}} .
\end{aligned}
$$

Substituting the value of $\alpha_{q}$ and omitting the higher order noise term $\sigma_{n}^{4}$, the above expression can be written as:

$$
\sigma_{\tilde{h}_{j, q}}^{2}=\frac{1}{\frac{P_{p, s} P_{p, r}}{\left(P_{p, r} \sigma_{h_{r, j}}^{2}+P_{p, s} \sigma_{h_{q, r}}^{2}\right) \sigma_{n}^{2}}+\frac{1}{\sigma_{h_{q, r}}^{2} \sigma_{h_{r, j}}^{2}}} .
$$

In the next section, we discuss the data transmission in an AF MWRN.

\section{DATA TRANSMisSiON}

At the end of pilot transmission at $t_{s}=i^{t h}$ time slot in the multiple access phase, the $i^{t h}$ and the $(i+1)^{t h}$ users simultaneously transmit the data signal $X_{i}$ and $X_{i+1}$, respectively. The relay receives the signal

$$
r_{i, i+1}=\sqrt{P_{d}} h_{i, r} X_{i}+\sqrt{P_{d}} h_{i+1, r} X_{i+1}+n_{1},
$$

where, $n_{1}$ is the zero mean complex AWGN at the relay with noise variance $\sigma_{n}^{2}$ per dimension.

In the broadcast phase, at the end of pilot transmission, the relay amplifies the received signal with $\alpha_{d}$ and then retransmits

$$
Y_{i, i+1}=\alpha_{d}\left(\sqrt{P_{d}} h_{i, r} X_{i}+\sqrt{P_{d}} h_{i+1, r} X_{i+1}+n_{1}\right) .
$$

Here,

$$
\alpha_{d}=\sqrt{\frac{P_{r}}{P_{d}\left(\sigma_{h_{i, r}}^{2}+\sigma_{h_{i+1, r}}^{2}\right)+\sigma_{n}^{2}}}
$$

is chosen to maintain the power constraint at the relay. The relay calculates these coefficients for each user pair and broadcasts them before re-transmission of the network coded signal.

The $j^{t h}$ user $(j \in[1, L])$ receives the signal

$$
\hat{Y}_{i, i+1}=h_{r, j} Y_{i, i+1}+n_{2},
$$

where $n_{2}$ is the zero mean complex AWGN at the $j^{\text {th }}$ user with noise variance $\sigma_{n}^{2}$ per dimension.

\section{A. Signal Extraction}

Finally, the $i^{t h}(i \in[1, L])$ user obtains the signals of all the other users by self interference cancelation. At first, it extracts the message of the $(i+1)^{t h}$ user (for $i<L$ ) in the downward extraction process and the $(i-1)^{t h}$ user (for $i>1)$ in the upward extraction process. Then the $i^{t h}$ user can extract the signal of the $m^{t h}$ user, where $i+1<m<L$ and $1<m<i-1$, in the downward and the upward signal extraction processes, respectively.

To extract the signals of the $(i \pm 1)^{t h}$ user (where, $i \pm 1$ denotes the user whose message is decoded by subtracting the $i^{\text {th }}$ user's self interference, in the downward and in the upward extraction process, respectively), the $i^{t h}$ user subtracts its own signal multiplied by $\alpha_{d} \sqrt{P_{d}} \hat{h}_{i, i}$. Thus, it obtains

$$
\begin{aligned}
\hat{X}_{i \pm 1} & =h_{r, i} Y_{i, i \pm 1}-\alpha_{d} \sqrt{P_{d}} \hat{h}_{i, i} X_{i}+n_{2} \\
& =\alpha_{d} \sqrt{P_{d}} \hat{h}_{i, i \pm 1} X_{i \pm 1}+n_{i \pm 1},
\end{aligned}
$$

Here, $n_{i \pm 1}$ denotes the noise terms present in the extracted signal of the $(i \pm 1)^{t h}$ user's signal. The noise terms in $n_{i \pm 1}$ is given as:

$$
\begin{aligned}
n_{i \pm 1}= & \alpha_{d} \sqrt{P_{d}} \tilde{h}_{i, i} X_{i}+\alpha_{d} \sqrt{P_{d}} \tilde{h}_{i, i \pm 1} \tilde{h}_{i, r} X_{i \pm 1}+\alpha_{d} \hat{h}_{r, i} n_{1} \\
& +\alpha_{d} \tilde{h}_{r, i} n_{1}+n_{2} .
\end{aligned}
$$

where, the first term indicates self-interference that cannot be completely cancelled out due to imperfect channel estimation, the second term is a component of the desired signal that is lost due to channel estimation error and the last three terms indicate complex AWGN noises.

Similarly, the $i^{t h}$ user extracts the message of the $m^{\text {th }}$ user. In the downward extraction process, the $i^{t h}$ user subtracts the extracted signal of the $(m-1)^{t h}$ user to obtain:

$$
\begin{aligned}
\hat{X}_{m} & =h_{r, i} Y_{m-1, m}-\alpha_{d} \sqrt{P_{d}} \hat{h}_{i, m-1} \hat{X}_{m-1}+n_{2} \\
& =\alpha_{d} \sqrt{P_{d}} \hat{h}_{i, m} X_{m}+n_{m},
\end{aligned}
$$

where, $n_{m}$ denotes the noise terms present in the extracted signal of the $m^{t h}$ user and is given as:

$$
\begin{aligned}
n_{m} & =\alpha_{d} \sqrt{P_{d}} \tilde{h}_{i, m-1} X_{m-1}+\alpha_{d} \sqrt{P_{d}} \tilde{h}_{i, m} X_{m}+\alpha_{d} \hat{h}_{r, i} n_{1} \\
& +\alpha_{d} \tilde{h}_{r, i} n_{1}+n_{2} .
\end{aligned}
$$

Here, we consider that at high SNR, $\hat{X}_{m-1}=X_{m-1}$, i.e., the individual error probabilities are zero and thus, no error propagation occurs. Similarly, in the upward extraction process, the $i^{t h}$ user subtracts the extracted signal of the $(m+1)^{t h}$ user to obtain the $m^{t h}$ user's signal and (13) and (14) would be applicable even in this case with the subscript $m-1$ replaced with $m+1$.

\section{Sum Rate Analysis}

The sum rate indicates the maximum throughput of the system. For an AF MWRN, the sum rate can be defined as the sum of the achievable rates of all users for a complete round of information exchange. In this section, we investigate the sum rate of an AF MWRN with imperfect CSI. First, we obtain the expression of the signal to noise ratio (SNR) at the $i^{t h}$ user. The SNR of the $(i \pm 1)^{t h}$ user's signal received at the $i^{\text {th }}$ user can be expressed by:

$$
\gamma_{i \pm 1}=\frac{\alpha_{d}^{2} P_{d} \sigma_{h_{r, i}}^{2} \sigma_{h_{i \pm 1, r}}^{2}}{N_{i \pm 1}}
$$

where, the numerator represents the power of the signal terms in (11), assuming that $\left|\hat{h}_{i, i \pm 1}\right|^{2}$ can be replaced by $\left|h_{i, i \pm 1}\right|^{2}$ for a tractable analysis and $N_{i \pm 1}$ represents the power of the noise terms in (12). That is,

$$
\begin{aligned}
N_{i \pm 1}= & \alpha_{d}^{2} P_{d} \sigma_{\tilde{h}_{i, i}}^{2}+\alpha_{d}^{2} P_{d} \sigma_{\tilde{h}_{i, i \pm 1}}^{2}+\alpha_{d}^{2} P_{d} \sigma_{h_{r, i}}^{2} \sigma_{n}^{2}+ \\
& \alpha_{d}^{2} P_{d} \sigma_{\tilde{h}_{r, i}}^{2} \sigma_{n}^{2}+\sigma_{n}^{2}
\end{aligned}
$$


Substituting the value of $\alpha_{d}$ from (9) and omitting the higher order noise terms, the SNR in (15) becomes:

$$
\gamma_{i \pm 1}=\frac{P_{r} P_{d} \sigma_{h_{r, i}}^{2} \sigma_{h_{i \pm 1, r}}^{2}}{N_{i \pm 1}^{\prime}}
$$

where

$$
\begin{gathered}
N_{i \pm 1}^{\prime}=P_{r} P_{d} \sigma_{\tilde{h}_{i, i}}^{2}+P_{r} P_{d} \sigma_{\tilde{h}_{i, i \pm 1}}^{2}+\left(P_{d}+P_{r}\right) \sigma_{h_{r, i}}^{2} \sigma_{n}^{2}+ \\
P_{d} \sigma_{h_{i \pm 1, r}}^{2} \sigma_{n}^{2}
\end{gathered}
$$

Similarly, the SNR of the $m^{\text {th }}$ user's signal received at the $i^{t h}$ user can be obtained from (13) by substituting $\alpha_{d}=$ $\sqrt{\frac{P_{r}}{P_{d}\left(\sigma_{h_{m, r}}^{2}+\sigma_{h_{m \pm 1, r}}^{2}\right)+\sigma_{n}^{2}}}$, where, $m \pm 1$ corresponds to upward and downward extraction processes, respectively. Thus,

$$
\gamma_{m}=\frac{P_{r} P_{d} \sigma_{h_{r, i}}^{2} \sigma_{h_{m, r}}^{2}}{N_{m}^{\prime}}
$$

where, $N_{m}^{\prime}=P_{r} P_{d} \sigma_{\tilde{h}_{i, m}}^{2}+P_{r} P_{d} \sigma_{\tilde{h}_{i, m \pm 1}}^{2}+P_{r} \sigma_{h_{r, i}}^{2} \sigma_{n}^{2}+$ $P_{d} \sigma_{h_{m, r}}^{2} \sigma_{n}^{2}+P_{d} \sigma_{h_{m \pm 1, r}}^{2} \sigma_{n}^{2}$ denotes the variance of the noise terms in (14). Note that the SNR expressions in (17) and (19) have similar form, apart from the fact that the denominator in (17) includes the interference components from the $i^{\text {th }}$ and the $(i \pm 1)^{t h}$ users, whereas, the denominator in (19) includes components from the $i^{t h}, m^{t h}$ and the $(m \pm 1)^{t h}$ users.

Now, in an AF MWRN, the maximum achievable rate at the $(k-1)^{t h}$ time slot is upper bounded by [4]:

$$
R_{k-1} \leq \frac{1}{2} \log \left(1+\gamma_{k}\right)
$$

where, $\gamma_{k}$ denotes the SNR of the $k^{t h}(k \neq i)$ user received at the $i^{t h}$ user, given by (17) and (19) and $\log ($.$) denotes$ logarithm to the base two.

Then substituting (17) and (19) in (20), adding the maximum achievable rates at all the time slots and after some algebraic manipulations, we can obtain the sum rate for symmetric traffic as:

$$
R_{s}=\frac{1}{2(L-1)} \sum_{k=1, k \neq i}^{L} \log \left(1+\frac{P_{r} P_{d} \sigma_{h_{r, i}}^{2} \sigma_{h_{k, r}}^{2}}{N_{k}^{\prime}}\right)
$$

\section{Power Allocation}

In this section, we obtain the power allocation coefficients in terms of users' statistical CSI for the pilot and the data signal powers of users and the relay to optimize the achievable sum rate of the network. Since, the average power per signal is $P$, the total energy for one pair of message exchange between a user pair is $(2 T+4) P T^{\prime}(T$ data transmission blocks from the two users and $T$ data transmission blocks from the relay, 1 pilot transmission block from each user and 2 pilot transmission blocks from the relay), as shown in Fig. 2. Since, the energy is conserved during this process, we can write

$$
2 P_{p, s} T^{\prime}+P_{d} T T^{\prime}+2 P_{p, r} T^{\prime}+P_{r} T T^{\prime}=(2 T+4) P T^{\prime} .
$$

From (17)-(21), we can see that the sum rate is maximized

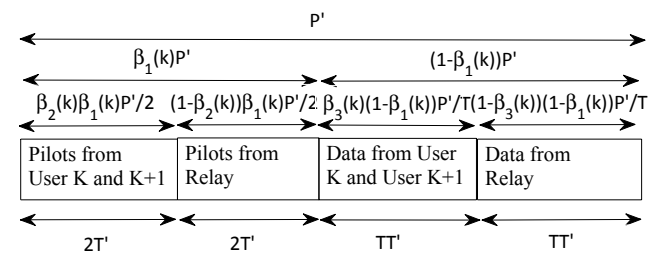

Fig. 2. Transmission structure for the pilot and data signals from the users and the relay at the $k^{t h}(k \in[1, L-1])$ time slot. Here, $P^{\prime}=\frac{(2 T+4)}{2} P$ denotes the total power during one pair of message exchange, $T$ represents the number of transmission blocks in the data signal and $T^{\prime}$ represents the duration of each transmission block. The optimum power allocation coefficients are shown at the top of the transmission blocks.

when the achievable rate at each time slot is maximized. Since, both the users in a user pair are allocated with the same transmission power at each time slot, optimizing the achievable rate at each time slot, ensures optimum performance for both the users. Further, from (20), it can be identified that the achievable rate at each time slot is maximized when the denominators of the SNRs in (17) and (19) are minimized. Thus, the optimum power allocation problem for a MWRN can be posed as allocating the average power to users' and the relay's pilot and data signals, such that the denominator in (21) is minimized, that is:

$$
\begin{aligned}
& \min _{P_{p, s}, P_{p, r}, P_{d}, P_{r}} f_{k} \\
& \text { s.t. } \quad P_{p, s}+P_{p, r}+P_{d}+P_{r}=P
\end{aligned}
$$

where, $f_{k}$ denotes the objective function at the $k^{\text {th }}$ time slot $(k \in[1, L-1]) . f_{k}$ can be expressed as a function of the $k^{t h}$ and the $(k+1)^{t h}$ users' statistical CSI and given by (24) at the top of the next page.

We assume that at the $k^{\text {th }}$ time slot, a fraction $\beta_{1}(k)$ of the total power is allocated to the pilot signals. We also assume that at the same time slot, $\beta_{2}(k)$ fraction of the pilot signal power and $\beta_{3}(k)$ fraction of the data signal power are allocated to the users. These power allocation coefficients are illustrated in Fig. 2 and can be represented as:

$$
\begin{aligned}
P_{p, s} & =\beta_{2}(k) \beta_{1}(k) \frac{(2 T+4) P}{2}, \\
P_{p, r} & =\left(1-\beta_{2}(k)\right) \beta_{1}(k) \frac{(2 T+4) P}{2}, \\
P_{d} & =\beta_{3}(k)\left(1-\beta_{1}(k)\right) \frac{(2 T+4) P}{T}, \\
P_{r} & =\left(1-\beta_{3}(k)\right)\left(1-\beta_{1}(k)\right) \frac{(2 T+4) P}{T} .
\end{aligned}
$$

Now, substituting (25) in (24), the optimization problem in (23) is solved to obtain the optimum power allocation coefficients $\beta_{1}(k), \beta_{2}(k)$ and $\beta_{3}(k)$ as in the following theorems.

Theorem 1: The optimum fraction of pilot signal power and 


$$
f_{k}= \begin{cases}\sigma_{\tilde{h}_{i, k}}^{2}+\sigma_{\tilde{h}_{i, k \pm 1}}^{2}+\frac{\sigma_{h_{r, i}}^{2} \sigma_{n}^{2}}{P_{d}}+\frac{\sigma_{h_{k, r}, r}^{2} \sigma_{n}^{2}}{P_{r}}+\frac{\sigma_{h_{k \pm 1, r}}^{2} \sigma_{n}^{2}}{P_{r}} & k \neq i \pm 1 \\ \sigma_{\tilde{h}_{i, i}}^{2}+\sigma_{\tilde{h}_{i, k}}^{2}+\frac{\sigma_{h_{r, i}}^{2} \sigma_{n}^{2}}{P_{d}}+\frac{\sigma_{h_{r, i}}^{2} \sigma_{n}^{2}}{P_{r}}+\frac{\sigma_{h_{k, r}}^{2} \sigma_{n}^{2}}{P_{r}} & k=i \pm 1\end{cases}
$$

data signal power, allocated to users, can be given by:

$$
\beta_{2}(k)=\beta_{3}(k)= \begin{cases}\frac{\sigma_{h_{i, r}}}{\sigma_{h_{i, r}}+\sqrt{\sigma_{h_{k, r}}^{2}+\sigma_{h_{k \pm 1, r}}^{2}}} & k \neq i \pm 1 \\ \frac{\sigma_{h_{i, r}}}{\sigma_{h_{i, r}}+\sqrt{\sigma_{h_{i, r}}^{2}+\sigma_{h_{i \pm 1, r}}^{2}}} & k=i \pm 1\end{cases}
$$

Proof: see Appendix A.

Theorem 2: The optimum fraction of the total power allocated to the pilot signals, can be given by:

$$
\beta_{1}(k)=\frac{C_{2}-\sqrt{C_{2}^{2}-4 C_{1} C_{2}}}{2 C_{1}}
$$

where, the coefficients are given by: $C_{1}=A_{1} \beta^{2}(k)(1-$ $\left.\beta^{2}(k)\right) P^{\prime 2}\left(2-\frac{T}{2}\right), C_{2}=2 A_{1} \beta(k)(1-\beta(k)) P^{\prime}(2 \beta(k)(1-$ $\left.\beta(k)) P^{\prime}+\frac{A_{1} A_{2} T}{2}\right), C_{3}=2 A_{1} \beta^{2}(k)\left(1-\beta^{2}(k)\right) P^{\prime 2}-\frac{A_{1}^{3} T A_{2}^{2}}{2}$, $\beta(k)=\beta_{2}(k)=\beta_{3}(k), A_{1}=\left((1-\beta(k)) \sigma_{h_{r, i}}^{2}+\right.$ $\left.2 \beta(k) \sigma_{h_{a v g}}^{2}\right) \sigma_{n}^{2}, A_{2}=\frac{1}{\sigma_{h_{r, i}}^{2} \sigma_{h_{a v g}}^{2}}$ and $\sigma_{h_{a v g}}^{2}=\frac{\sigma_{h_{k, r}}^{2}+\sigma_{h_{k \pm 1, r}}^{2}}{2}$. Proof: see Appendix B.

Remark 1: From (26), it can be noted that when the $i^{\text {th }}$ (receiving) user has large average channel gain, less power is allocated to the relay and more to the $k^{t h}$ and the $(k \pm 1)^{t h}$ (transmitting) users and vice versa.

Remark 2: It can be noted that the optimum power allocation coefficients $\beta_{1}(k), \beta_{2}(k)$ and $\beta_{3}(k)$ are functions of long terms statistical CSI of the users' channels. Thus, the computation complexity of these coefficients at the relay would be quite simple, which could enable a faster operation compared to different complex optimization techniques.

\section{RESULTS}

In this section, we provide numerical simulation results to verify the analysis on the optimum power allocation of an AF MWRN. We perform Monte Carlo simulation to obtain the sum rates over different channel realizations for $L=10$ user MWRN, where each user transmits one pilot signal and a data packet of $T=1000$ signals. Following [4], the average channel gain for the $j^{\text {th }}$ users is modeled by $\sigma_{h_{j, r}}^{2}=\left(1 /\left(d_{j} / d_{0}\right)\right)^{\nu}$, where $d_{0}$ is the reference distance, $d_{j}$ is the distance between the $j^{t h}$ user and the relay which is assumed to be uniformly randomly distributed between 0 and $d_{0}$, and $\nu$ is the path loss exponent, which is assumed to be 3 . The SNR is defined as $\frac{P^{\prime}}{N_{0}}$ and the $i^{t h}$ user is assumed to be the decoding user.

Fig. 3 shows the optimum power allocation coefficients at the $k^{\text {th }}$ time slot for two sets of channel conditions of the users: (i) $d_{i}=0.1 d_{0}, d_{k \pm 1}=0.9 d_{0}$ (the $i^{t h}$ user has good channel conditions) and (ii) $d_{i}=0.9 d_{0}, d_{k \pm 1}=0.1 d_{0}$ (the $i^{\text {th }}$ user has poor channel conditions). For all the above cases, we have considered the distance of the $k^{\text {th }}$ user as $d_{0}$ which

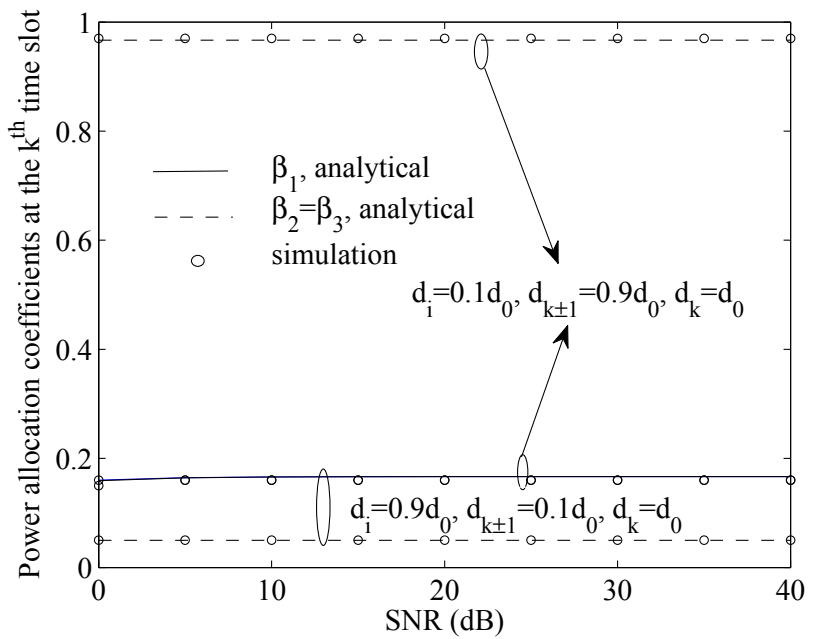

Fig. 3. Optimum power allocation coefficients at the $k^{t h}(k \in[1, L-1])$ time slot in an $L=10$-user AF MWRN for different channel conditions.

corresponds to the worst possible channel conditions. The distances of other users (except the $i^{t h}, k^{t h}$ and the $(k \pm 1)^{t h}$ users) are randomly distributed between 0 and $d_{0}$. In this figure, the analytical results for the optimum coefficient $\beta_{1}(k)$ (see (27)) and $\beta_{2}(k)=\beta_{3}(k)$ (see (26)) are verified through numerical search. Here, for the channel conditions in (i), we can see that a larger fraction of the pilot power and the data power are allocated to the transmitting users $\left((k \pm 1)^{t h}\right.$ and $\left.k^{t h}\right)$, compared to the relay (i.e., larger $\beta_{2}(k)$ and $\beta_{3}(k)$ ), as explained in Remark 1. However, the power allocated to the pilot signals (i.e., $\beta_{1}(k)$ ) remains the same for both the channel conditions. This is due to the fact that the parameter $A_{1}$ (see after Theorem 2) is nearly the same for both the channel conditions, because for both the cases, $d_{i}=d_{0}-d_{k \pm 1}$. As a result, the coefficient $\beta_{1}(k)$ remains unchanged for the set of distances $\left\{d_{i}, d_{k \pm 1}\right\}$ that satisfies $d_{i}=d_{0}-d_{k \pm 1}$.

Fig. 4 shows that optimum power allocation gives higher sum rate for both the cases when (i) $10 \%$ users have distances below $0.1 d_{0}$ (most of the users have bad channel conditions) and (ii) $90 \%$ users have distances below $0.1 d_{0}$ (most of the users have good channel conditions). It can be seen from the figure that when most of the users experience poor channel conditions, the performance degradation for optimum power allocation is smaller compared to that for equal power allocation. Thus, optimum power allocation makes the network performance less vulnerable towards degradation in the overall channel conditions. It can be seen that when most of the users experience good and bad channel conditions, optimum power allocation saves the power by $7 \mathrm{~dB}$ and $9 \mathrm{~dB}$, respectively, to achieve the same sum rate as equal power allocation. 


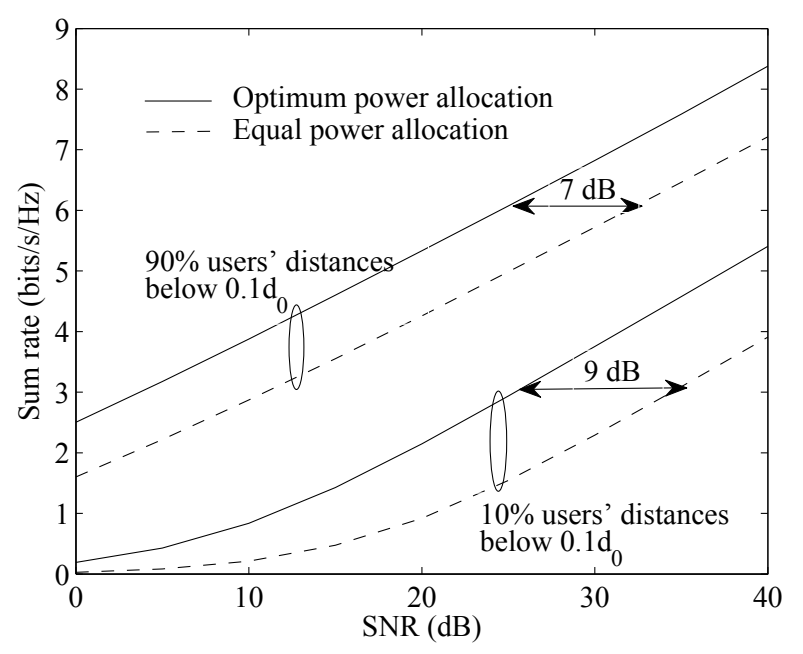

Fig. 4. Sum rate when $10 \%$ and $90 \%$ users' distances are below $0.1 d_{0}$ in an $L=10$-user AF MWRN.

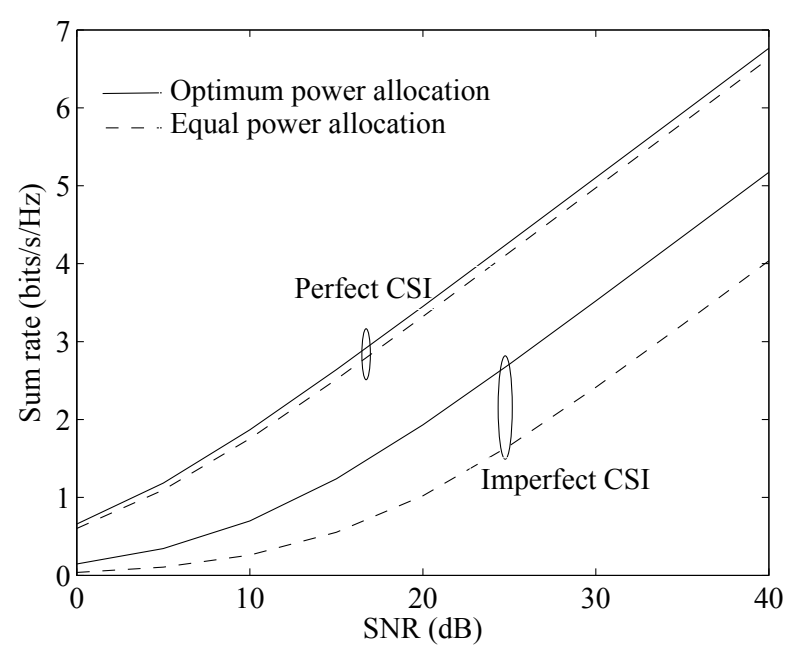

Fig. 5. Sum rate with imperfect and perfect CSI for an $L=10$-user AF MWRN.

Fig. 5 shows the impact of optimum power allocation on the achievable sum rate in the presence of imperfect and perfect channel estimation. It can be seen from the figure that when there is no channel estimation error, the optimum power allocation problem simplifies to conventional water filling power allocation. In this case, the degree of improvement in the sum rate provided by optimum power allocation is relatively small. However, in the presence of imperfect channel estimation, optimum power allocation provides a large improvement in the sum rate compared to equal power allocation, e.g., we can see from the figure that for equal power allocation, when perfect CSI is not available, the sum rate drops by $2.7 \mathrm{bits} / \mathrm{s} / \mathrm{Hz}$ at 40 $\mathrm{dB}$ SNR. On the other hand, for optimum power allocation, the drop is about $1.2 \mathrm{bits} / \mathrm{s} / \mathrm{Hz}$ at $40 \mathrm{~dB}$ SNR. Thus, optimum power allocation helps to make the system performance more robust to imperfect CSI.

\section{CONCLUSIONS}

In this paper, we have investigated optimum power allocation to maximize the achievable sum rate in AF MWRNs assuming that only long term statistical CSI is available at the users and the relay. We have shown that for optimum power allocation, the sum rate performance of an AF MWRN becomes less vulnerable towards the degradation in the overall channel conditions. In addition, we observe that optimum power allocation requires $7-9 \mathrm{~dB}$ less SNR to achieve the same sum rate obtained by equal power allocation depending on the users' channel conditions. We have also shown that optimum power allocation is more robust to imperfect CSI, compared to equal power allocation.

\section{APPENDIX A \\ PROOF OF THEOREM 1}

First, we consider the $k \neq(i \pm 1)^{t h}$ time slot. The objective function in this case can be written as, $f_{k}=f_{p, k}+f_{d, k}$, where, $f_{p, k}=\sigma_{\tilde{h}_{i, k}}^{2}+\sigma_{\tilde{h}_{i, k \pm 1}}^{2}$ and $f_{d, k}=\frac{\sigma_{h_{r, i}}^{2} \sigma_{n}^{2}}{P_{d}}+\frac{\sigma_{h_{k, r}}^{2} \sigma_{n}^{2}}{P_{r}}+$ $\frac{\sigma_{h_{k \pm 1, r}}^{2} \sigma_{n}^{2}}{P_{r}}$ represent the functions involving the pilot signal power and the data signal power, respectively. Using (25), we can write $f_{p, k}$ and $f_{d, k}$ in terms of the power allocation coefficients, as:

$$
\begin{aligned}
f_{p, k}= & \frac{1}{\frac{(2 T+4) P \beta_{2}(k) \beta_{1}(k)\left(1-\beta_{2}(k)\right)}{2\left(\left(1-\beta_{2}(k)\right) \sigma_{h_{r, i}}^{2}+\beta_{2}(k) \sigma_{h_{k, r}}^{2}\right) \sigma_{n}^{2}}+\frac{1}{\sigma_{h_{k, r}}^{2} \sigma_{h_{r, i}}^{2}}}+ \\
& \frac{1}{\frac{(2 T+4) P \beta_{2}(k) \beta_{1}(k)\left(1-\beta_{2}(k)\right)}{2\left(\left(1-\beta_{2}(k)\right) \sigma_{h_{r, i}}^{2}+\beta_{2}(k) \sigma_{h_{k \pm 1, r}}^{2}\right) \sigma_{n}^{2}}+\frac{1}{\sigma_{h_{k \pm 1, r}}^{2} \sigma_{h_{r, i}}^{2}}}
\end{aligned}
$$

and

$f_{d, k}=\left(\frac{\sigma_{h_{r, i}}^{2}}{\beta_{3}(k)}+\frac{\sigma_{h_{k, r}}^{2}+\sigma_{h_{k \pm 1, r}}^{2}}{\left(1-\beta_{3}(k)\right)}\right) \frac{T \sigma_{n}^{2}}{\left(1-\beta_{1}(k)\right)(2 T+4) P}$.

Now, setting $\frac{d f_{d, k}}{d \beta_{3}(k)}=0$ yields

$\beta_{3}^{2}(k)\left(\sigma_{h_{r, i}}^{2}-\left(\sigma_{h_{k, r}}^{2}+\sigma_{h_{k \pm 1, r}}^{2}\right)\right)-2 \beta_{3}(k) \sigma_{h_{r, i}}^{2}+\sigma_{h_{r, i}}^{2}=0$.

Solving the above equation leads to the optimum value of $\beta_{3}(k)$ as in (26). Similarly, it can be shown that setting $\frac{d f_{k}}{d \beta_{2}(k)}=0$, gives $\beta_{2}(k)=\beta_{3}(k)$, which completes the proof. For $k=(i \pm 1)^{t h}$ time slot, the proof can be completed in a similar way, with $\sigma_{h_{k, r}}^{2}$ replaced by $\sigma_{h_{i, r}}^{2}$ in the above equations.

\section{APPENDIX B}

PROOF OF THEOREM 2

If we set $\beta_{2}(k)=\beta_{3}(k)=\beta(k)$ and $\frac{(2 T+4)}{2} P=P^{\prime}$, $P_{p, s}, P_{p, r}, P_{d}$ and $P_{r}$ can be substituted by $\beta_{1}(k) \beta(k) P^{\prime},(1-$ $\beta(k)) \beta_{1}(k) P^{\prime}, 2 \beta(k)\left(1-\beta_{1}(k)\right) P^{\prime} / T$ and $2(1-\beta(k))(1-$ 
$\left.\beta_{1}(k)\right) P^{\prime} / T$, respectively. Then (24) can be written as:

$$
\begin{gathered}
f=\frac{1}{\frac{\beta(k) \beta_{1}(k)(1-\beta(k)) P^{\prime}}{\left((1-\beta(k)) \sigma_{h_{r, i}}^{2}+\beta(k) \sigma_{h_{k, r}}^{2}\right) \sigma_{n}^{2}}+\frac{1}{\sigma_{h_{r, i}}^{2} \sigma_{h_{k, r}}^{2}}}+ \\
\frac{1}{\frac{\beta(k) \beta_{1}(k)(1-\beta(k)) P^{\prime}}{\left((1-\beta(k)) \sigma_{h_{r, i}}^{2}+\beta(k) \sigma_{h_{k \pm 1, r}}^{2}\right) \sigma_{n}^{2}}+\frac{1}{\sigma_{h_{r, i}}^{2} \sigma_{h_{k \pm 1, r}}^{2}}}+ \\
\left(\frac{\sigma_{h_{r, i}}^{2}}{2 \beta(k)}+\frac{\sigma_{h_{k, r}}^{2}+\sigma_{h_{k \pm 1, r}}^{2}}{2(1-\beta(k))}\right) \frac{\sigma_{n}^{2} T}{\left(1-\beta_{1}(k)\right) P^{\prime}} .
\end{gathered}
$$

Now, setting $\frac{d f_{d, k}}{d \beta_{1}(k)}=0$ leads to the optimum $\beta_{1}(k)$. However, the exact solution of this equation would be very complicated. To simplify the analysis, we set $\sigma_{h_{k, r}}^{2}=\sigma_{h_{k \pm 1, r}}^{2}=$ $\frac{\sigma_{h_{k, r}}^{2}+\sigma_{h_{k \pm 1, r}}^{2}}{2}=\sigma_{h_{a v g}}^{2}$, which can quantify the average impact of the transmitting users' channel conditions on the optimum solution of $\beta_{1}(k)$. Thus, (30) can be simplified to

$f=\frac{2}{\frac{\beta_{1}(k) \beta(k)(1-\beta(k)) P^{\prime}}{A_{1}}+A_{2}}+\frac{A_{1} T}{2\left(1-\beta_{1}(k)\right) \beta(k)(1-\beta(k)) P^{\prime}}$,

where, $A_{1}=\left((1-\beta(k)) \sigma_{h_{r, i}}^{2}+2 \beta(k) \sigma_{h_{a v g}}^{2}\right) \sigma_{n}^{2}$ and $A_{2}=$ $\frac{1}{\sigma_{h_{r, i}}^{2} \sigma_{h_{a v g}}^{2}}$. Now, differentiating (31) with respect to $\beta_{1}(k)$ and equating to zero leads to a quadratic equation, the root of which gives the optimal $\beta_{1}(k)$ in (27), such that $\beta_{1}(k)<1$. Thus, the proof is completed.

\section{REFERENCES}

[1] D. Gündüz, A. Yener, A. Goldsmith, and H. V. Poor, "The multi-way relay channel," IEEE Trans. Inf. Theory, vol. 59, no. 1, pp. 51-63, Jan. 2013.

[2] L. Ong, S. J. Johnson, and C. M. Kellett, "An optimal coding strategy for the binary multi-way relay channel," IEEE Commun. Lett., vol. 14, no. 4 , pp. $330-332$, Apr. 2010

[3] G. Amarasuriya, C. Tellambura, and M. Ardakani, "Performance analysis of pairwise amplify-and-forward multi-way relay networks," IEEE Wireless Commun. Lett., vol. 1, no. 5, pp. 524-527, Oct. 2012.

[4] —, "Multi-way MIMO amplify-and-forward relay networks with zero-forcing transmission," IEEE Trans. Commun., vol. 61, no. 12, pp. 4847-4863, Dec. 2013.

[5] S. N. Islam, S. Durrani, and P. Sadeghi, "A novel user pairing scheme for functional decode-and-forward multi-way relay network," Physical Communication, Sep. 2014, submitted. [Online]. Available: http://arxiv.org/abs/1402.6422v3

[6] S. Islam and P. Sadeghi, "Joint decoding: Extracting the correlation among user pairs in a multi-way relay channel," in Proc. IEEE PIMRC, Sep. 2012, pp. 54-59.

[7] S. Katti, S. Gollakota, and D. Katabi, "Embracing wireless interference: Analog network coding," in Proc. ACM SIGCOMM, 2007, pp. 397-408

[8] R. H. Y. Louie, Y. Li, and B. Vucetic, "Practical physical layer network coding for two-way relay channels: performance analysis and comparison," IEEE Trans. Wireless Commun., vol. 9, no. 2, pp. 764-777, Feb. 2010.

[9] T. Cui, T. Ho, and J. Kliewer, "Memoryless relay strategies for two-way relay channels," IEEE Trans. Commun., vol. 57, no. 10, pp. 3132-3143, Oct. 2009.

[10] S. N. Islam, P. Sadeghi, and S. Durrani, "Error performance analysis of DF and AF multi-way relay networks with BPSK modulation," IET Commun., vol. 7, no. 15, pp. 1605-1616, Oct. 2013.

[11] H. Degenhardt, Y. Rong, and A. Klein, "Non-regenerative multi-way relaying: Combining the gains of network coding and joint processing," IEEE Trans. Wireless Commun., vol. 12, no. 11, pp. 5692-5703, Nov. 2013
[12] M. Wang, F. Wang, and Z. Zhong, "Queue-aware power allocation for multi-way relay networks," in Proc. IEEE ICC, Jun. 2012, pp. 55735577.

[13] S. Wang, Q. Song, X. Wang, and A. Jamalipour, "Rate and power adaptation for analog network coding," IEEE Trans. Veh. Technol., vol. 60 , no. 5, pp. 2302-2313, 2011.

[14] M. Pischella and D. Le Ruyet, "Optimal power allocation for the twoway relay channel with data rate fairness," IEEE Commun. Lett., vol. 15 no. 9, pp. 959-961, Sept. 2011.

[15] S. Talwar, Y. Jing, and S. Shahbazpanahi, "Joint relay selection and power allocation for two-way relay networks," IEEE Signal Process. Lett., vol. 18, no. 2, pp. 91-94, Feb. 2011.

[16] B. Jiang, F. Gao, X. Gao, and A. Nallanathan, "Channel estimation and training design for two-way relay networks with power allocation," IEEE Trans. Wireless Commun., vol. 9, no. 6, pp. 2022-2032, June 2010.

[17] L. Song, "Relay selection for two-way relaying with amplify-andforward protocols," IEEE Trans. Veh. Technol., vol. 60, no. 4, pp. 1954 1959, May 2011.

[18] M. Chen and A. Yener, "Power allocation for multi-access two-way relaying," in Proc. IEEE ICC, Jun. 2009, pp. 1-5.

[19] — , "Power allocation for F/TDMA multiuser two-way relay networks," IEEE Trans. Wireless Commun., vol. 9, no. 2, pp. 546-551, Feb. 2010.

[20] F. Tabataba, P. Sadeghi, C. Hucher, and M. Pakravan, "Impact of channel estimation errors and power allocation on analog network coding and routing in two-way relaying," IEEE Trans. Veh. Technol., vol. 61, no. 7, pp. 3223-3239, Sept. 2012.

[21] G. Kramer, M. Gastpar, and P. Gupta, "Cooperative strategies and capacity theorems for relay networks," IEEE Transactions on Information Theory, vol. 51, no. 9, pp. 3037-3063, Sep. 2005.

[22] F. Tabataba, P. Sadeghi, and M. Pakravan, "Outage probability and power allocation of amplify and forward relaying with channel estimation errors," IEEE Trans. Wireless Commun., vol. 10, no. 1, pp. 124-134, Jan. 2011. 